\title{
Sodium Bicarbonate—A Potent Ergogenic Aid?
}

\author{
Jose Shelton ${ }^{1}$, Gideon V. Praveen Kumar ${ }^{2}$ \\ ${ }^{1}$ Victoria University, Melbourne, Australia; ${ }^{2}$ School of Biosciences and Technology, VIT University, Vellore, India. \\ Email: \{josenoel2008, gideonpraveenkumar\}@gmail.com \\ Received May $30^{\text {th }}, 2010$; revised July $4^{\text {th }}, 2010$; accepted July $7^{\text {th }}, 2010$.
}

\begin{abstract}
This report aims to look at the role of Sodium bicarbonate as a potent Ergogenic aid and its role in improving the performance of athletes. It includes the mechanism of action of sodium bicarbonate during high-intensity exercise. The report also shows the various types of athletes who can be benefited from sodium bicarbonate loading, evidences for improvement in performance, conflicting evidences, recommended dosages and side-effects for bicarbonate loading.
\end{abstract}

Keywords: Sodium Bicarbonate, Ergogenic Aid, High-Intensity Exercise, Anaerobic Glycolytic System

\section{Introduction}

An ergogenic aid is defined as any substance, food, chemical, or training method that helps the body work harder and perform better. Ergogenic aids are any external influences that can positively affect physical or mental performance [1]. These include mechanical aids, pharmacological aids, physiological aids, nutritional aids, and psychological aids. Athletes at all levels of competitions are constantly striving for a means to obtain a leading edge over their opponents and many of them use ergogenic aids to improve their energy and performance [2]. Ergogenic aids may directly influence the physiological capacity of a particular body system thereby improving performance, remove psychological constraints which impact performance, and increase the speed of recovery from training and competition [3].

The term Ergogenic means "to produce work". Nutritional ergogenic aids are dietary manipulations that can increase physical power or energy production, enhance mental strength, or provide a mechanical edge and thereby improve sport performance [4]. Dietary manipulations encompass 3 major efforts: alteration of food choices, addition of macronutrients for specific uses in sports and exercise, addition of micronutrients for specific uses in sports and exercise [3].

\section{Energy System for High-Intensity Exercise}

Of the 3 primary energy pathways, the anaerobic glycolytic system provides fuel sources, primarily carbohydrates, for high-intensity exercises (exercises lasting between 20 seconds up to about 90 seconds). Anaerobic glycolysis provides the primary fuel source for exercise of near-maximal intensity lasting longer than about 30 seconds. High-intensity exercises (anaerobic work) involve the breakdown of carbohydrates (muscle glycogen). As the glycogen stores are rapidly depleted, there is a resulting increase in hydrogen ion $\left(\mathrm{H}^{+}\right)$concentrations and lactic acid in both the muscle and blood. It is this increase in $\mathrm{H}^{+}$concentration (drop in $\mathrm{pH}$ ) that causes a progressive increase in the acidity of the muscle cells (intracellular environment). The increase in $\mathrm{H}^{+}$produces, among other reactions, an inhibition of calcium release from the sarcoplasmic reticulum and an inhibition of the interaction between actin and myosin [5]. The drop in $\mathrm{pH}$ as a result of lactic acid accumulation is thought to inhibit the resynthesis of adenosine triphosphate (ATP) as well as inhibit muscle contraction. This in turn results in muscular fatigue defined as a decrease in force production in the presence of increased perception of effort and an inability to maintain high exercise intensities [6,7].

\section{Sodium Bicarbonate}

Sodium bicarbonate or sodium hydrogen carbonate is the chemical compound with the formula $\mathrm{NaHCO}_{3}$. Sodium bicarbonate is a white solid that is crystalline but often appears as a fine powder. It has a slight alkaline taste resembling that of washing soda (sodium carbonate). It is a component of the mineral natron and is found dissolved in many mineral springs. The natural mineral form is known as nahcolite. It is also produced artificially. Since it has long been known and is widely used, the salt has many related names such as baking soda, bread soda, cooking soda, bicarbonate of soda. Colloquially, its name is shortened to sodium bicarb. 


\section{Role of Sodium Bicarbonate during High-Intensity Exercise}

During high-intensity exercise, muscles keep up with the demand for energy by converting some carbohydrate to lactic acid. A build-up of acid inside the muscle cells is one of the factors responsible for fatigue. This system's total capacity is limited by the progressive increase in acidity within the muscles caused by the accumulation of lactate and $\mathrm{H}^{+}$ions. Increased acidity ultimately inhibits energy transfer and the ability of the muscles to contract, leading to a decrease in exercise performance. Reducing the build-up of acid should reduce fatigue and allow the athlete to go faster or further. The body's defences against an increase in acidity are the bicarbonate "buffers", which help to neutralize the acid produced by intense exercise. The natural bicarbonate supply, part of the body's buffering system, provides a rapid first line of defence against this increased acidity.

\section{Acting Mechanism}

Sodium bicarbonate is an alkalising agent and therefore reduces the acidity of the blood (known as a buffering action). By buffering acidity in the blood, bicarbonate may be able to draw more of the acid produced within the muscle cells out into the blood and thus reduce the level of acidity within the muscle cells themselves. This in turn could delay the onset of fatigue [8]. Studies [9,10] have confirmed that increased extracellular $\mathrm{pH}$ and higher bicarbonate raise the $\mathrm{H}^{+}$and lactic acid efflux from active muscles. This is due to an increase in the activity of the Lactic acid $/ \mathrm{H}^{+}$co-transporter, which becomes more active as the intracellular/extracellular $\mathrm{H}^{+}$ gradient increases, during contraction as well as during recovery. It has been suggested that this mechanism causes a decrease in muscular fatigue, delaying the decrease in $\mathrm{pH}$ level and leading to a greater contractile capacity of the muscular tissue involved by means of enhanced muscle glycolytic ATP production. It has been proven that all of these metabolic perturbations imply a shift in muscle metabolism toward anaerobic energy production, which is especially advantageous during high-intensity exercise [5].

Taking a sufficient quantity of sodium bicarbonate (baking soda) before high-intensity event makes the muscles and blood less acidic during the event and can enhance physical performance [11].

\section{Athletes Benefiting from Sodium Bicarbonate Loading}

Athletes have been practising "soda loading" or "bicarbonate loading" for over 70 years in an attempt to delay the onset of muscular fatigue during prolonged anaerobic exercise. The specific athletes who might stand to benefit from bicarb supplementation are those who typically compete in events that last between 1 and 7 minutes, i.e. $400 \mathrm{~m}-1500 \mathrm{~m}$ running, $100 \mathrm{~m}-400 \mathrm{~m}$ swimming, sprint cycling, kayaking, rowing and canoeing events at intensities that fall between 80 and $125 \%$ of peak maximal oxygen uptake, and many team sports with their repeated nature of high intensity exercise. All these events stress the anaerobic glycolysis system significantly and produce a lot of acidity. Sports that are dependent on repeated anaerobic bursts may also benefit from bicarbonate loading $[6,8]$.

\section{Evidences for Improvement in Performance}

A study done by McNaughton et al. [9], among moderately trained female athletes, showed significant improvement in both work and power output, during high intensity exercise of 60 second duration. The ingestion of sodium bicarbonate in the experimental trial had the desired effect of raising blood bicarbonate levels by $60 \%$ above the resting bicarbonate value.

According to Stellingwerff [12], a meta-analysis of 29 studies on the performance effects of sodium bicarbonate, featuring predominately untrained individuals, found that bicarbonate supplementation resulted in a performance effect that was 0.44 standard deviations better than in the control trial. An improvement of 0.44 of the standard deviation would bring the 2006 average men's $800 \mathrm{~m}$ Golden League time of 1:46.36 down to 1:45.52, which is a worthwhile improvement. In summary, most data suggest that the ingestion of $0.3 \mathrm{~g} / \mathrm{kg}$ body weight of sodium bicarbonate administered in solution approximately 1-2 hours before exercise offers a small, but significant, effect on middle-distance race performance.

In one study [7] designed to simulate athletic competition, trained non-elite middle-distance runners performed a simulated $800 \mathrm{~m}$ race. In the alkalotic condition, they ran almost $3 \mathrm{~s}$ faster than in the placebo or control trials. Another report by Maughn et al. [13] indicated similar improvements (3-4 s) over a distance of $1500 \mathrm{~m}$ in runners who completed simulated races in about 4 min $15 \mathrm{~s}$. Although these effects on performance might appear small, they are of considerable significance to the athlete, for whom an improvement of even a fraction of a second in these events is considered to be a major achievement. In a study by VanMontfoort et al. [10], ingestion of sodium bicarbonate was found to increase the sprint performance in elite male athletes compared to citrate, lactate and chloride ingestion.

Studies by McNaughton et al. [14] have also found that sodium bicarbonate can be used as an ergogenic aid to offset the fatigue process in high-intensity, competitive cycle ergometry of 1 hour duration. 


\section{Conflicting Evidence}

However, even though many of the studies have shown that sodium bicarbonate administration modifies the blood acid-base balance, its effects on performance are not always positive [5]. For instance, no improvements have been registered following 90 seconds of maximal cycle exercise by untrained men [15].

No differences were found between the experimental or placebo trials in female cyclists pedalling at $95 \% \mathrm{VO} 2$ max [9]. This suggested that bicarbonate buffering does not improve performance in female athletes during repeated bouts of high intensity exercise.

The reasons for the conflicting effects are not altogether clear, but are at least in part due to variations in the intensity and duration of the exercise tests used, the nature of the exercise task, the dosage of bicarbonate administered and the time delay between bicarbonate administration and the beginning of the exercise test (i.e. the amount of metabolic alkalosis induced). Performance has been monitored over exercise durations ranging from a few seconds to more than 1 hour, and during continuous, incremental and intermittent dynamic exercise as well as during sustained isometric contractions [13].

There is no clear pattern of exercise duration between those studies where a positive effect was observed and those where no effect was seen. In most studies, a dose of $0.3 \mathrm{~g}$ of sodium bicarbonate $/ \mathrm{kg}$ of body weight has been used to induce alkalosis, and this has usually been administered orally in solution or in capsule form. Such a dose has usually resulted in an increase of $4-5 \mathrm{mmol} . \mathrm{L}^{-1}$ in the plasma buffer base 2-3 hours after administration, although the time-course of changes in acid-base status was not carefully followed in most of these studies [16].

Those studies in which high-intensity and short-term exercises (less than 2 minutes) were used, and in which the doses of sodium bicarbonate given were lower than $0.3 \mathrm{~g} / \mathrm{kg}$ body weight, did not generally produce an enhancement of performance, which might be due to the use of an insufficient dose or due to the short duration of the effort [5]. Therefore, it has been suggested that exercises lasting less than 1 minute may not be of sufficient duration for the glycolytic metabolism to activate completely, in that the capacity of the intracellular buffer is exceeded and a positive gradient between the intracellular and extracellular medium is established. The main reason for the lack in performance improvement may consequently be due more to the exercise duration than to the doses used.

\section{Recommended Dosage}

Many studies indicate an effective dose of $300 \mathrm{mg}$ of sodium bicarbonate per kg body weight taken 1-2 hours prior to short-term maximal, high intensity performance lasting $60 \mathrm{sec}$ shows $30 \%$ improvement in performance $[6,11]$. Sodium bicarbonate appears to be safe when taken in the recommended dose of $140 \mathrm{mg} /$ pound of body weight. For a 150 pound athlete this translates to a dose of 5 teaspoons of baking soda that provides $21,000 \mathrm{mg}$ or $21 \mathrm{~g}$ of bicarbonate. Safety should not be confused with side effects. Baking soda should be consumed with plenty of water, (eg. a litre or more), when taken 1 or 2 hours prior to exercise [4].

\section{Side Effects}

There are of course potential problems of severe alkalosis associated with the use of increased doses of bicarbonate. Additionally side effects like vomiting, gastrointestinal discomfort, bloating, and diarrhea may occur particularly if sufficient water is not taken with sodium bicarbonate. Vomiting and diarrhea are frequently reported as a result of ingestion of even relatively small doses of bicarbonate, and this may limit any attempt to improve athletic performance by this method, certainly among those individuals susceptible to gastrointestinal problems. There have been reports of athletes using this intervention, which is not prohibited by the rules of the sport, being unable to compete because of the severity of these symptoms. Although unpleasant and to some extent debilitating, these effects are not serious and there are no long term adverse consequences [17].

\section{Acute vs Chronic Loading}

Given that some individuals exhibit urgent gastrointestinal distress with bicarbonate, such as vomiting and diarrhoea, it is important for athletes to experiment with bicarbonate in training that features daily consecutive races, since much of the gastrointestinal distress seems to occur after a race (semi-finals), which could limit performance in any subsequent race (finals) [12].

Studies $[18,19]$ have shown more favourable gastrointestinal tolerance effects after chronic multiday bicarbonate supplementation as compared to acute pre-exercise single-dose administration. Performance in highintensity exercise may be enhanced for a full 2 days after cessation of chronic bicarbonate supplementation which might alleviate many of the severe gastrointestinal sideeffects found with acute bicarbonate loading.

Not withstanding these results, more research is needed to show performance efficacy for chronic bicarbonate ingestion protocols in elite athletes, and to better elucidate the dosing and time-course effects between the cessation of dosing and exercise performance testing.

\section{Conclusions}

In conclusion, there are sufficient data to suggest that sodium bicarbonate can be used as a nutritional ergogenic aid or dietary supplement for improving performance in short term, high intensity exercise, provided it is taken in the recommended dosage of $300 \mathrm{mg}(0.3 \mathrm{~g}) / \mathrm{kilogram}$ 
body weight. Continued use of bicarbonate may help athletes become less susceptible to the side effects and may give even larger improvements in performance.

\section{Further Research}

Since the time elapsed from the ingestion of bicarbonate to the beginning of exercise (time of absorption) varies considerably, until further research can clarify the time course and cessation of dosing that that can lead to performance enhancement, the individual athlete is advised to experiment in training to judge their own case. The athlete needs to discover not only the potential for performance improvement, but also the likelihood of unwanted side-effects. Further research is also needed to find out the longest duration of endurance exercise that benefits from the use of sodium bicarbonate.

\section{REFERENCES}

[1] D. M. Ahrendt, "Ergogenic Aids: Counselling the Athlete," American Family Physician, Vol. 63, No. 5, 2001, pp. 913-922.

[2] J. R. Berning and S. N. Steen, "Sports Nutrition for the 90's: The Health Professional's Handbook," Aspen Publishers, Gaithersburg, 1991.

[3] I. Wolinsky and J. A. Driskell, "Nutritional Ergogenic Aids," CRC Press, Boca Raton, 2004.

[4] M. Ryan, "Sports Nutrition for Endurance Athletes," Velo Press, Boulder, 2002.

[5] B. Requena, M. Zabala, P. Padial and B. Feriche, "Sodium Bicarbonate and Sodium Citrate: Ergogenic Aids," Journal of Strength and Conditioning Research, Vol. 19, No. 1, 2005, pp. 213-224.

[6] E. Applegate, "Effective Nutritional Ergogenic Aids," International Journal of Sports Nutrition, Vol. 9, No. 2, 1999, pp. 229-239.

[7] R. J. Maughn, L. M. Burke and E. F. Coyle, "Food, Nutrition and Sports Performance II-The International Olympic Committee Consensus on Sports Nutrition," Routledge Publishing, Cornwall, 2004.

[8] L. Burke and V. Deakin, "Clinical Sports Nutrition," 2nd Edition, McGraw-Hill Book Company, Sydney, 2000, p. 478.

[9] L. R. McNaughton, S. Ford and C. Newbold, "Effect of Sodium Bicarbonate Ingestion on High Intensity Exercise in Moderately Trained Women," Journal of Strength and
Conditioning Research, Vol. 11, No. 2, 1997, pp. 98-102.

[10] M. C. E. VanMontfoort, L. VanDieren, W. G. Hopkins and J. P. Shearman, "Effects of Ingestion of Bicarbonate, Citrate, Lactate, and Chloride on Sprint Running," Medicine and Science in Sports and Exercise, Vol. 36, No. 7, 2004, pp. 1239-1243.

[11] L. R. McNaughton, B. Dalton, J. Tarr and D. Buck, "Neutralize Acid to Enhance Performance," Sportscience Training \& Technology, 1997. http://www.sportsci.org/traintech/buffer/lrm.htm

[12] T. Stellingwerff, M. K. Boit and P. T. Res, "Nutritional Strategies to Optimize Training and Racing in MiddleDistance Athletes," Journal of Sports Sciences, Vol. 25, No. S1, 2007, pp. S17-S28.

[13] R. J. Maughn, D. S. King and T. Lea, "|Dietary Supplements," Journal of Sports Sciences, Vol. 22, No. 1, 2004, pp. 95-113.

[14] L. McNaughton, B. Dalton and G. Palmer, "Sodium Bicarbonate can be Used as an Ergogenic Aid in High-Intensity, Competitive Cycle Ergometry of $1 \mathrm{~h} \mathrm{Du}$ ration," European Journal of Applied Physiology, Vol. 80, No. 1, 1999, pp. 64-69.

[15] J. O. Marx, S. E. Gordon, N. H. Vos, B. C. Nindl, A. L. Gomez, J. S. Volek, J. Pedro, N. Ratamess, R. U. Newton, D. N. French, M. R. Rubin, K. Hakkinen, J. William and W. J. Kraemer, "Effect of Alkalosis on Plasma Epinephrine Responses to High Intensity Cycle Exercise in Humans," European Journal of Applied Physiology, Vol. 87, No. 1, 2002, pp. 72-77.

[16] R. J. Maughan, "Nutritional Ergogenic Aids and Exercise Performance," Nutrition Research Reviews, Vol. 12, No. 2, 1999, pp. 255-280.

[17] R. J. Maughan and L. M. Burke, "Handbook of Sports Medicine and Science: Sports Nutrition," Blackwell Publishing, Cornwall, 2002.

[18] I. I. Douroudos, I. G. Fatouros, V. Gourgoulis, A. Z. Jamurtas, T. Tsitsios, A. Hatzinikolaou, K. Margonis, K. Mavromatidis and K. Taxildaris, "Dose-Related Effects of Prolonged $\mathrm{NaHCO}_{3}$ Ingestion during High-Intensity Exercise," Medicine and Science in Sports and Exercise, Vol. 38, No. 10, 2006, pp. 1746-1753.

[19] J. Edge, D. Bishop and C. Goodman, "Effects of Chronic $\mathrm{NaHCO}_{3}$ Ingestion during Interval Training on Changes to Muscle Buffer Capacity, Metabolism, and Short-Term Endurance Performance," Journal of Applied Physiology, Vol. 101, 2006, pp. 918-925. 